\title{
Achieving a good crystal system for crystallographic X-ray fragment screening
}

Patrick M Collins a , Alice Douangamath ${ }^{a}$, Romain Talon ${ }^{a}$, Alexandre Dias a , José Brandão-Neto ${ }^{a}$, Tobias Krojer $^{b}$, Frank von Delft ${ }^{a, b, c, 1}$

${ }^{a}$ Diamond Light Source, Harwell Science and Innovation Campus, Didcot, OX11 ODE, UK

b Structural Genomics Consortium, Nuffield Department of Medicine, University of Oxford, Oxford, OX3 7DQ, UK

' Department of Biochemistry, University of Johannesburg, Aukland Park, 2006, South Africa

Corresponding author: e-mail address: frank.von-delft@diamond.ac.uk

Keywords: Fragment Screening, XChem, Protein Crystallisation, X-ray crystallography, Diamond Light Source, 104-1, Structural Genomics Consortium

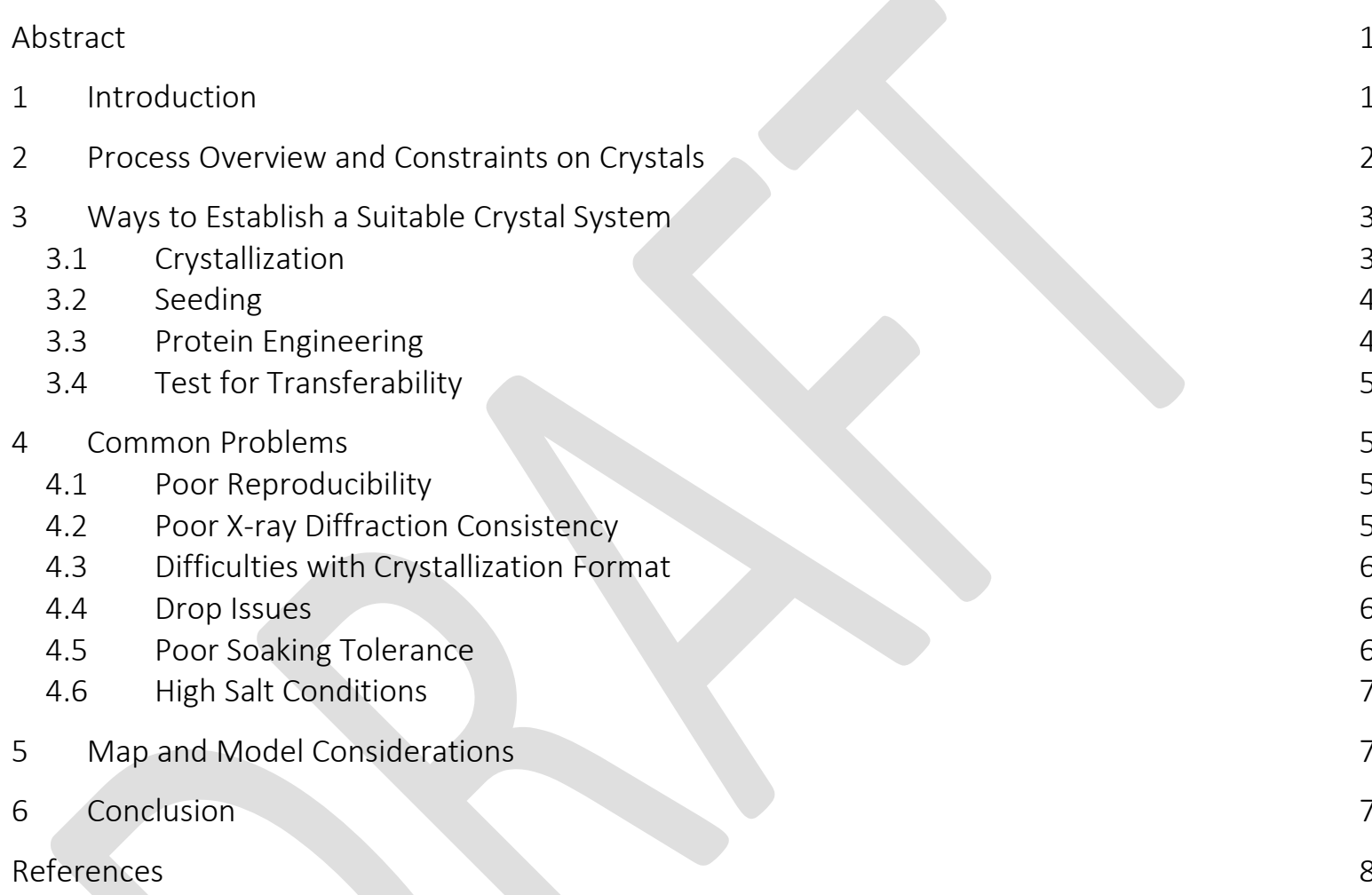

\section{Abstract}

The XChem facility at Diamond Light Source offers fragment screening by X-ray crystallography as a general access user program. The main advantage of $X$-ray crystallography as a primary fragment screen is that it yields directly the location and pose of the fragment hits, whether within pockets of interest or merely on surface sites: this is the key information for structure-based design and for enabling synthesis of follow-up molecules. Extensive streamlining of the screening experiment at $X C h e m$ has engendered a very active user programme that is generating large amounts of data: in 2017, 36 academic and industry groups generated 35,000 datasets of uniquely soaked crystals. It has also generated a large number of learnings concerning the main remaining bottleneck, namely obtaining a suitable crystal system that will support a successful fragment screen. Here we discuss the practicalities of generating screen-ready crystals that have useful electron density maps, and how to ensure they will be successfully reproduced and usable at a facility outside the home lab.

\section{Introduction}

Many biophysical methods have been described for the primary fragment screen, including both ligand- and protein-observed NMR spectroscopy (Harner, Frank, \& Fesik, 2013), surface plasmon resonance (SPR) (Navratilova \& Hopkins, 2010), X-ray crystallography (Hartshorn et al., 2005), to name 
only a few. Invariably, however, knowledge of the fragment binding mode is crucial, and co-crystal structures of bound hits are generally sought, in order to initiate iterative structure-based design and synthesis cycles. X-ray crystallography is, along with NMR, the oldest primary screen, and in principle represents the gold standard, since it provides direct structural readout for follow-up design, and is unmatched in sensitivity (Patel, Bauman, \& Arnold, 2014). However, the logistical overheads of collecting X-ray diffraction data from 100s to 1000 s of crystals have historically made it prohibitive for most groups. More recently, though, advances in synchrotron science and automation have reduced these overheads, enabling the development of high-throughput X-ray diffraction data collection. Taking advantage of this potential throughput, however, including for experiments such as fragment screening, places much higher demands on crystal production.

There are two basic approaches to obtaining protein-fragment complexes, namely co-crystallization or soaking (Hassell et al., 2007). Co-crystallization involves mixing of the free protein in solution with the small molecule prior to crystallization, which allows the ligand to bind to the protein without any crystal lattice constraints, and in principle allowing for more conformational freedom of the protein. This is the method of choice when a protein complex with a specific ligand is required. High throughput methods for co-crystallization with small molecules have been described previously (Gelin et al., 2015). The drawback is that the addition of various small molecules in co-crystallization tends to lower crystallization success rates, and induce larger variations in resolution and crystal form, placing a greater burden on downstream analysis in the high-throughput setting. In this approach, false negatives arise from the failure of the protein to crystallize, or the crystals selecting for protein molecules with no compound bound.

Crystal soaking is simpler: compound is added, dissolved or pure, directly to the crystallization drop, and it subsequently diffuses through the crystal solvent channels to reach the binding pockets (Danley, 2006). This approach yields diffracting crystals more reliably, since the many crystals required can be grown from the same condition; X-ray diffraction also tends to be more consistent. Instead, false negatives arise from failure of the compound to reach the binding pocket, or the protein conformation being incompatible with binding, the latter leading either to empty pockets, or crystals dissolving upon compound binding due to lattice disruption. Low compound solubility undermines both cocrystallization and soaking.

The methods described here are tailored to screening by soaking, as this is the approach implemented in the XChem facility at Diamond Light Source, which is a platform for high-throughput fragment screening by crystal structure. The soaking protocol itself has been described previously (Collins et al., 2017), and comprises fast crystal soaking using acoustic droplet ejection techniques. Additionally, the platform relies on machine-assisted crystal harvesting, automated X-ray diffraction data collection, and streamlined data processing (Krojer et al., 2017) with enhanced fragment hit detection (Pearce et al., 2017). As the first ever facility supporting such an experiment, it has enabled the generation of $1000 \mathrm{~s}$ of protein-fragment complexes for large numbers of industrial and academic users (Delbart et al., 2018; Foley et al., 2017; Harding et al., 2017; Keedy et al., 2018; McIntyre et al., 2017).

The high throughput nature of crystal-based fragment screening places specific demands on protein crystals that tend not to apply to traditional structure solution projects. The hands-on support required by the nature of the XChem facility has led to the accumulation of a large amount of experience of getting problematic crystal systems to work, and these lessons and common problems are the focus of the rest of the chapter.

\section{Process Overview and Constraints on Crystals}

To perform the screen, the 100s of crystals need to be on site; unsurprisingly, therefore, how to ensure this is the case dominates the early conversation between users and XChem staff for every experiment. Users close to the synchrotron may find it easiest to grow crystals at home lab and bring them to Diamond for the experiment. In contrast, for users further afield or without crystallization robotics in-house, crystallization is performed on-site, either by the attending user, or by XChem staff. In this case, the crystallization protocol must be smoothly transferable from lab to facility, requiring a level of robustness of protocol not usually addressed, and further discussed below.

The experiment performed at the facility itself proceeds as follows: 
1. Crystal plates are imaged in standard crystal plate imagers (Formulatrix). Crystal drop images are analyzed using TeXRank (Ng, Dekker, Kroemer, Osborne, \& von Delft, 2014), and suitable crystal wells are selected and marked with target coordinates for acoustic dispensing of fragments.

2. Fragments from a source library in dimethyl sulfoxide (DMSO) solvent are acoustically transferred (Labcyte) to the targeted crystal drop locations, and the crystals allowed to soak (Collins et al., 2017).

3. Crystals are manually harvested into cryo-loops and frozen in liquid nitrogen, the process robotically assisted with a motorized microscope stage (Shifter; Oxford Lab Technologies).

4. X-ray diffraction data are collected in automated, unattended mode at Diamond beamlines (i04-1), with crystals aligned by centering with fast X-ray rastering.

5. Data are automatically integrated, scaled and electron density maps calculated by molecular substitution.

6. Electron density maps are analyzed using PanDDA (Pearce et al., 2017) for detection and modeling of bound fragments.

7. Models are refined and prepared for deposition to the PDB, with all calculations managed and tracked using XChemExplorer (Krojer et al., 2017).

This process is deliberately highly flexible, since most crystal systems have peculiarities that must be accommodated. Nevertheless, the likelihood of overall success is greatly enhanced if as many as possible of the common constraints can be dealt with - and this generally is feasible, with some focused effort.

- Reproducibility of crystallization: many 100 s of crystals must grow in a specific crystallization format (for XChem: the SBS-format 3-lens SwissCi plates), with ideal drop volumes of 100-600 nL. Drops with volumes smaller than $100 \mathrm{~nL}$ tend to be more difficult to mount crystals from due to rapid evaporation of the drop, and drops over $600 \mathrm{~nL}$ require so much compound that the speed of acoustic dispensing (500 $\mathrm{nL} / \mathrm{sec})$ becomes limiting.

- Crystal size and robustness: if crystals are large enough (>30 $\mu \mathrm{m})$, they become reliable to manipulate during harvesting; and when robust, they can tolerate both fast manual harvesting the high solvent concentrations in the drop after dissolved compound is added.

- Consistency of diffraction: to improve robustness of data analysis, crystals should have consistent $X$-ray diffraction, ideally with resolution to better than $2.5 \AA$. Any problems encountered during data analysis slow down the overall experiment enormously, and are worth avoiding.

- Suitable molecular packing: while accessibility of the binding site is a basic physical constraint, high quality of electron density in the regions of interest and low conformational heterogeneity greatly simply analysis of the data.

\section{Ways to Establish a Suitable Crystal System}

It is a common beginner's mistake to consider one's crystal system robust, merely because the first structure was achieved quite straightforwardly: in reality, the requirements are far more stringent. The description below therefore covers how to approach the challenge of generating large numbers of suitable crystals, as well as how to ensure that these crystals will also in fact be available at the remote facility where the fragment screen is to be performed.

\subsection{Crystallization}

Quality of crystals is always the dominant consideration when selecting between crystallization conditions, should a choice of conditions be available. Beyond that, the nature of the precipitant is important: polyethylene glycol (PEG) conditions are preferable over high salt conditions, since the latter tend to reduce fragment solubility and result in heavy compound precipitation/crystallization on addition to the drop, thereby reducing the effective compound concentration (see also section 4.6 below). Volatile solvents such as isopropanol are inconvenient, as they lead to rapid evaporation of the crystal drop and cause difficulties during crystal mounting (see section 4.4 below). Finally, the $\mathrm{pH}$ should be as close to physiological as possible, to maximize the likelihood of the binding sites having their relevant protonation state.

To provide the best chances of consistent X-ray diffraction after crystal soaking, it is important that all crystallization drops across a plate have identical components. Variations in equilibrated drop 
volume (for example, from variable reservoir:protein ratios) will result in variable final compound and solvent concentrations after soaking, which can result in inconsistent $\mathrm{X}$-ray diffraction for sensitive systems. Graduation of components across a plate (such as $\mathrm{pH}$ or precipitant concentration gradients) should also be avoided for optimal consistency.

Subtle changes to the crystallization protocol can profoundly change the crystal system's behavior. Therefore, if the crystallization protocol changes in any detail (construct, crystallization components, drop volumes, purification batch, etc.), it is vital that control experiments are run to confirm that behavior remains as expected, including solvent tolerance, before embarking on the large fragment screen. This includes crystal age, with older crystals frequently having dramatic deterioration of diffraction consistency.

Robotic crystallization is essential to achieve consistent small drop volumes and provide the throughput necessary for the many crystallization plates usually required. The XChem facility relies on the resources of the crystallization facility in the neighboring Research Complex at Harwell, with Mosquito liquid handlers (TTP Labtech) available for nanodrop crystallization. The standard experiment is performed in the 96-well, 3-lens crystallization plates (Swiss Cl), containing 20 uL of reservoir solution and $150 \mathrm{~nL}$ crystal drops in a 1:1 ratio of protein to reservoir ( $75 \mathrm{~nL}$ each).

\subsection{Seeding}

Seeding is an extremely effective tool in the quest for consistent crystallization; it is also the trick most studiously ignored by newcomers to screening, until they have first failed to achieve reproducibility for several rounds of seedless optimization. It is now the single strongest recommendation tendered by XChem staff, being the closest thing to a magic bullet available.

In particular, it offers the necessary close control of the nucleation process for ensuring that a sufficient number of crystals can be grown for a large screen. Our seeding protocol follows that described in detail by (D'Arcy, Bergfors, Cowan-Jacob, \& Marsh, 2014), with seed stock aliquots frozen at $-80^{\circ} \mathrm{C}$ after preparation. By preparing and freezing a particular seed stock in aliquots, a specific seed dilution can be established that tends to reproduce the initial seeding trial significantly better than preparing fresh seed stocks prior to each round of crystallization.

Seeding is also a route for establishing alternative crystal forms: matrix seeding (Shaw Stewart, Kolek, Briggs, Chayen, \& Baldock, 2011) has yielded some remarkable successes, though close attention to the details appears to be crucial, which may be why there are also many anecdotes of it "not working".

\subsection{Protein Engineering}

It is rare that extensive exploration and optimization of crystallization conditions will convert poor, sporadic crystals into a strong, reliable system. Protein engineering provides a far more reliable if labor intensive route, a wisdom long since widely adopted by experienced practitioners.

Structural genomics initiatives in particular have established useful ground rules for discovering wellbehaved constructs. At the SGC, the standard approach is to generate many $(>10)$ truncated or mutated versions of the same protein up front and in parallel, to establish immediately whether any constructs have sufficient levels of protein expression to achieve solubility and purity to test for crystallization (Gileadi et al., 2008; Savitsky et al., 2010).

Similarly, having access to multiple starting points can be pivotal to a successful fragment screen. Not only does crystal quality vary, but availability of different crystal forms permits alternatives to be explored if the leading crystal system proves to be refractory further down the screening process, such as having poor solvent tolerance or an occluded binding site (discussed below). The detailed methods outlined by Gileadi et al. (2008) can be set up in any protein production lab and helps generate more options when embarking on a fragment screening campaign.

Another way to help coax a well-behaved protein to crystallize or change crystal form is to explore surface site mutations that introduce so-called "crystallization epitopes" that correlate with crystallization propensity (Price et al., 2009). In our hands, this approach has yielded many new crystal forms (manuscript in preparation). 


\subsection{Test for Transferability}

It is critical to establish that the crystal system established in the home lab can be reproduced at another facility. The main guideline is to minimize the number of processes that must be performed at the remote facility: thus ideally, all components, including the protein, seed stocks, crystallization cocktail, all at the correct concentrations, can be frozen for transport, to be thawed again at the destination for use. The exact dispensing protocol will also have been established at the home lab, ideally using the same robotics, and very thoroughly documented. This way, the only processes that need occur at the facility are the thawing of relevant components, and robotic preparation of the crystallization plate.

Importantly, the freeze/thaw cycle should be tested exhaustively at the home lab, to verify that reproducibility is maintained. And finally, the packaging should contain copious amounts of dry ice, at least $20 \mathrm{~kg}$ for a trans-Atlantic shipment, to provide for the inevitable delays at customs or mistakes by the courier company.

\section{Common Problems}

Here we summarize common problems and how they can be addressed.

\subsection{Poor Reproducibility}

Often, only a small fraction of drops will contain any crystals, or crystals of sufficient size/morphology. The following options can drastically improve reproducibility and productivity of each plate:

1. Seeding, as discussed above (section 3.2).

2. Exploring a different area of the phase diagram, such as a 2-4 fold increase in protein concentration (if possible), coupled with an equal fold (or more) decrease in precipitant concentration.

3. A broad optimization screen around the crystallization condition, exploring a wide $\mathrm{pH}$ and salt concentration range.

4. Ensuring that the purification protocol is yielding consistent protein quality, with gel filtration as the final step in purification.

Slow appearance of protein crystals (weeks or months) indicates a process that is not driven by vapor diffusion, but instead a drift in $\mathrm{pH}$, oxidation or evaporation through the plastic: in these cases, looking for very different crystallization conditions will help. Protein degradation is also a very common reason, and often the clearest evidence is a marked difference between what was purified and what's visible in the structure. Then it is highly advisable to adjust the expression construct to match what was observed in the structure: this frequently transforms not only the rate of crystallization, but also the quality of the resulting crystals.

Excess nucleation or overly rapid crystal formation may be reduced by small amounts of cryoprotectants (such as glycerol) added to the reservoir, or by lower (or higher) temperatures. Showers of crystals can sometimes be rescued by placing crystal plates containing small crystals through a high temperature cycle, for example placing them at $37^{\circ} \mathrm{C}$ for a few hours/overnight before returning to the lower crystallization temperature.

Alternatively, if protein is plentiful and a few quality crystals do appear in each crystallization plate, a valid strategy is simply to prepare large numbers of crystal plates until the desired number of crystals is obtained. In the XChem lab, this slows down the overall soaking and harvesting process only marginally, while enabling a successful fragment screen.

\subsection{Poor X-ray Diffraction Consistency}

Confirmation of whether $X$-ray diffraction inconsistency is inherent to the crystal system rather than the crystals' sensitivity to soaking, mounting and freezing, can be confirmed by in situ or room temperature X-ray diffraction experiments. For inherently inconsistent crystals, the same points raised above for crystal reproducibility tend to be relevant, especially seeding. Likewise, it becomes important to use only freshly grown crystals, and assess carefully their decay time in the drop. Sometimes, crystal quality is related to crystal morphology, which requires an observant experimenter to notice; in such cases, more careful crystal selection would help, though often requiring larger numbers of crystallization plates. 
If soaking and mounting are the cause of inconsistent diffraction, common factors to check are:

1. Ensure that crystal drops are of consistent volume from the crystallization setup. If drop volumes are variable, but the same volume of solvent (e.g. DMSO) is added, the final solvent concentrations will vary and this could undermine consistency.

2. Ensure that drop ratios are the same. When setting up crystallization plates, it is common to vary the protein:reservoir ratios (e.g. 1:2, 1:1, 2:1 through the 3 sub-wells). The drops will achieve different final volumes after vapor diffusion, again resulting in varying solvent concentrations, as above.

3. Ensure that the crystallization condition is uniform across the plate. A precipitant or $\mathrm{pH}$ gradient may result in some crystals being more sensitive to the screening treatments or requiring greater cryoprotection.

4. If soaking using acoustic methods, ensure that targeting and solvent dispensing is occurring at the edges of the crystal drop, and away from crystals.

5. Check the crystal harvesting technique and ensure a fast and efficient plunge into liquid nitrogen with minimal time passing through the cold gas layer (keep mounting dewar topped up with fresh liquid nitrogen).

6. Dehydration could be a factor (see section 4.4 below).

7. Reduce the amount of solvent, and thus lower the fragment concentration.

Certainly, some natural and stochastic variation in data quality is to be expected across many hundreds of crystals, and therefore the real question to ask is whether the data obtained are of sufficient quality to clearly answer the scientific question. For fragment screening, the main requirement is identification of sufficient hits, in sufficiently clear poses, to progress with hit-to-lead chemistry. This is frequently possible even when attrition of diffraction through the screen was unusually high.

\subsection{Difficulties with Crystallization Format}

Sometimes converting from one crystallization format, such as hanging drop, to one compatible with the XChem facility (sitting drop), may result in large changes to the crystallization outcome, such as clear drops or heavy precipitate. Since the target protein has been shown to crystallize, it is often a matter of optimization (see Poor Reproducibility section above), with exploration of protein/precipitant concentrations and seeding often proving successful after 2-3 weeks of focused effort in advance of embarking on the visit to the screening facility.

\subsection{Drop Issues}

Minor problems with the crystallization drop that can interfere with crystal harvesting, such as a skin over the drop, are greatly amplified if the protocol requires many hundreds of crystals to be manually mounted. Skins that cover a drop can be removed manually with a loop or acupuncture needle, but can also sometimes be avoided by ensuring that fresh crystallization plates are used, trying larger or smaller drop volumes, or by adding a 1:1 equivalent volume of reservoir solution prior to soaking and harvesting.

Other problems that can affect crystal mounting are drops that rapidly dry out or result in showers of additional crystals appearing rapidly after unsealing of wells. Similar solutions to those for the skin can resolve such issues, especially the addition of additional reservoir solution, or setting up larger initial crystallization drops. Addition of reservoir prior to soaking can also help to prevent X-ray diffraction inconstancies due to crystals that are sensitive to dehydration. Additionally, when mounting crystals, minimizing the number of wells that are unsealed during mounting, even when using devices for assisted crystal harvesting (the Shifter in the XChem lab), can reduce the effects of dehydration for sensitive systems.

\subsection{Poor Soaking Tolerance}

Some crystals have very low tolerance to being soaked in the presence of fragment carrying solvents, such as DMSO. Such solvent sensitivity can be difficult to overcome in soaking experiments. Addition of reservoir solution (1:1 volume ratio) to crystal drop just prior to solvent addition for crystal soaking can help to stabilize sensitive crystals. Alternatively, a fast soaking scheme, like flash soaking $(<5$ seconds) may be required to minimize soaking times and enable crystal survival. 
While DMSO is the most common solvent in use in fragment work, alternative solvents such as ethylene glycol are considerably gentler and may help for sensitive crystal systems. This does mean repreparing the fragment library, but not prohibitively so, especially if it converts an intractable system to a tractable one. Exploration of alternative crystal forms or crystallization conditions is sometimes required to discover a solvent tolerant soaking system.

\subsection{High Salt Conditions}

As discussed above (section 3.1), high concentrations of salt in the crystallization condition may reduce small molecule solubility and consequently reduce the effective soaking concentration. There are a number of examples of successful fragment screens in high salt conditions, and with high hit rates. However, if a low hit rate is observed and a high salt condition is suspected as the cause, optimization of any existing PEG-based crystal hits may afford a low salt crystal form. Alternatively, matrix seeding using crystal seeds from the salt condition into PEG-based crystal screens may provide new hits (D'Arcy et al., 2014). Another alternative is to transfer crystals from the salt-based condition into osmotically matched PEG containing drops. An appropriate PEG condition may be discovered by adding a significant excess (2:1 v/v or larger) of PEG solutions of increasing concentration to a series of drops: one of these additions will maintain the osmotic pressure and thus allow the crystal to survive the change; and in an appropriately equipped facility, the series of crystals can be quickly tested for diffraction.

\section{Map and Model Considerations}

Certain aspects of the actual structure and corresponding electron density maps can also have a large influence on the success of the fragment screen. Diffraction resolution is the most obvious, and at resolutions poorer than $2.5 \AA$, the loss of detail in the maps makes it very hard to confidently assign details of binding for molecules as small as fragments. In addition, the soaking process itself invariably causes a loss of resolution, and therefore crystals really ought to diffract natively at least to $2.3 \AA$ if the screening experiment is to be painless.

Any other pathologies that affect map quality will also affect the ability to assign the weakly binding fragments unambiguously. Thus features like a large twin fraction, strong anisotropy, or translational non-crystallographic symmetry can cause complications. On the other hand, achieving consistent map quality and resolution greatly helps with downstream processing and map analysis using PanDDA (Pearce et al., 2017).

Crystal conditions that result in multiple space groups or crystal forms from the same condition are less problematic than they might appear, as they can be handled reasonably effectively by downstream processing using XChemExplorer (Krojer et al., 2017), as long as good appropriate input models are available for each crystal form. Nevertheless, this does constitute a complication that many XChem users work hard to avoid.

Finally, the binding site itself needs to be open to the solvent channels, and as far as possible from a crystal contact, as these often involve conformational adjustments that might propagate to the site of interest in subtle ways. It will also ideally not have side chains or stretches of backbone that occupy multiple conformations or are completely disordered: energetically, this increases the entropic penalty of binding; and practically, such variations are very hard to model and therefore produce artefacts. Sometimes a small trial screen of 100 fragments may reveal that fragments can after all access the binding site; more likely though it will show more clearly that a new crystal form will be needed.

\section{Conclusion}

Thanks to modern synchrotron technologies, crystal-based fragment screens are now not only accessible through user programs, but the work involved is now far less than that required in the home lab to achieve a suitable crystal system. Close attention to detail and rigorous experimental practice remain crucial, and certainly XChem users gain a very intensive understanding of the crystallogenic and biophysical behavior of their system, quite apart from the chemistry readout of the screening itself.

Overall, the best approach is to recruit the help of an experienced crystallographer that has herself dealt with the relevant problems before, particularly those working in industry. Serendipitously, the scale of the XChem operation means that the number of crystallographers with the requisite experience 
will continue to rise, allowing for a self-reinforcing dynamic in the community. It is also to be expected that this increasing competence will prove useful to challenges of crystallogenesis at the other end of the scale, namely the need for reproducible showers for serial crystallography approaches such as needed for X-ray Free Electron Laser (XFEL) experiments.

\section{References}

Collins, P. M., Ng, J. T., Talon, R., Nekrosiute, K., Krojer, T., Douangamath, A., . . von Delft, F. (2017). Gentle, fast and effective crystal soaking by acoustic dispensing. Acta Crystallogr D Struct Biol, 73(Pt 3), 246-255. doi:10.1107/S205979831700331X

D'Arcy, A., Bergfors, T., Cowan-Jacob, S. W., \& Marsh, M. (2014). Microseed matrix screening for optimization in protein crystallization: what have we learned? Acta Crystallogr F Struct Biol Commun, 70(Pt 9), 1117-1126. doi:10.1107/S2053230X14015507

Danley, D. E. (2006). Crystallization to obtain protein-ligand complexes for structure-aided drug design. Acta Crystallogr D Biol Crystallogr, 62(Pt 6), 569-575. doi:10.1107/S0907444906012601

Delbart, F., Brams, M., Gruss, F., Noppen, S., Peigneur, S., Boland, S., . . Ulens, C. (2018). An allosteric binding site of the alpha7 nicotinic acetylcholine receptor revealed in a humanized acetylcholine-binding protein. J Biol Chem, 293(7), 2534-2545. doi:10.1074/jbc.M117.815316

Foley, D. J., Craven, P. G. E., Collins, P. M., Doveston, R. G., Aimon, A., Talon, R., . . Nelson, A. (2017). Synthesis and Demonstration of the Biological Relevance of sp(3) -rich Scaffolds Distantly Related to Natural Product Frameworks. Chemistry, 23(60), 15227-15232. doi:10.1002/chem.201704169

Gelin, M., Delfosse, V., Allemand, F., Hoh, F., Sallaz-Damaz, Y., Pirocchi, M., . . Guichou, J.-F. (2015). Combining 'dry' co-crystallization and in situ diffraction to facilitate ligand screening by X-ray crystallography. Acta Crystallographica Section D, 71(8), 1777-1787. doi:doi:10.1107/S1399004715010342

Gileadi, O., Burgess-Brown, N. A., Colebrook, S. M., Berridge, G., Savitsky, P., Smee, C. E., ... Pantic, N. H. (2008). High throughput production of recombinant human proteins for crystallography. Methods Mol Biol, 426, 221-246. doi:10.1007/978-1-60327-058-8_14

Harding, R. J., Ferreira de Freitas, R., Collins, P., Franzoni, I., Ravichandran, M., Ouyang, H., . . . Arrowsmith, C. H. (2017). Small Molecule Antagonists of the Interaction between the Histone Deacetylase 6 Zinc-Finger Domain and Ubiquitin. J Med Chem, 60(21), 9090-9096. doi:10.1021/acs.jmedchem.7b00933

Harner, M. J., Frank, A. O., \& Fesik, S. W. (2013). Fragment-based drug discovery using NMR spectroscopy. J Biomol NMR, 56(2), 65-75. doi:10.1007/s10858-013-9740-z

Hartshorn, M. J., Murray, C. W., Cleasby, A., Frederickson, M., Tickle, I. J., \& Jhoti, H. (2005). Fragmentbased lead discovery using X-ray crystallography. J Med Chem, 48(2), 403-413. doi:10.1021/jm0495778

Hassell, A. M., An, G., Bledsoe, R. K., Bynum, J. M., Carter, H. L., 3rd, Deng, S. J., . . . Shewchuk, L. M. (2007). Crystallization of protein-ligand complexes. Acta Crystallogr D Biol Crystallogr, 63(Pt 1), 72-79. doi:10.1107/S0907444906047020

Keedy, D. A., Hill, Z. B., Biel, J. T., Kang, E., Rettenmaier, T. J., Brandao-Neto, J., . . Fraser, J. S. (2018). An expanded allosteric network in PTP1B by multitemperature crystallography, fragment screening, and covalent tethering. Elife, 7. doi:10.7554/eLife.36307

Krojer, T., Talon, R., Pearce, N., Collins, P., Douangamath, A., Brandao-Neto, J., . . . von Delft, F. (2017). The XChemExplorer graphical workflow tool for routine or large-scale protein-ligand structure determination. Acta Crystallogr D Struct Biol, 73(Pt 3), 267-278. doi:10.1107/S2059798316020234

Mclntyre, P. J., Collins, P. M., Vrzal, L., Birchall, K., Arnold, L. H., Mpamhanga, C., . . Bayliss, R. (2017). Characterization of Three Druggable Hot-Spots in the Aurora-A/TPX2 Interaction Using Biochemical, Biophysical, and Fragment-Based Approaches. ACS Chem Biol, 12(11), 2906-2914. doi:10.1021/acschembio.7b00537

Navratilova, I., \& Hopkins, A. L. (2010). Fragment screening by surface plasmon resonance. ACS Med Chem Lett, 1(1), 44-48. doi:10.1021/ml900002k 
Ng, J. T., Dekker, C., Kroemer, M., Osborne, M., \& von Delft, F. (2014). Using textons to rank crystallization droplets by the likely presence of crystals. Acta Crystallogr D Biol Crystallogr, 70(Pt 10), 2702-2718. doi:10.1107/S1399004714017581

Patel, D., Bauman, J. D., \& Arnold, E. (2014). Advantages of crystallographic fragment screening: functional and mechanistic insights from a powerful platform for efficient drug discovery. Prog Biophys Mol Biol, 116(2-3), 92-100. doi:10.1016/j.pbiomolbio.2014.08.004

Pearce, N. M., Krojer, T., Bradley, A. R., Collins, P., Nowak, R. P., Talon, R., . . von Delft, F. (2017). A multi-crystal method for extracting obscured crystallographic states from conventionally uninterpretable electron density. Nat Commun, 8, 15123. doi:10.1038/ncomms15123

Price, W. N., 2nd, Chen, Y., Handelman, S. K., Neely, H., Manor, P., Karlin, R., . . Hunt, J. F. (2009). Understanding the physical properties that control protein crystallization by analysis of largescale experimental data. Nat Biotechnol, 27(1), 51-57. doi:10.1038/nbt.1514

Savitsky, P., Bray, J., Cooper, C. D., Marsden, B. D., Mahajan, P., Burgess-Brown, N. A., \& Gileadi, O. (2010). High-throughput production of human proteins for crystallization: the SGC experience. J Struct Biol, 172(1), 3-13. doi:10.1016/j.jsb.2010.06.008

Shaw Stewart, P. D., Kolek, S. A., Briggs, R. A., Chayen, N. E., \& Baldock, P. F. M. (2011). Random Microseeding: A Theoretical and Practical Exploration of Seed Stability and Seeding Techniques for Successful Protein Crystallization. Crystal Growth \& Design, 11(8), 3432-3441. doi:10.1021/cg2001442 